\title{
IL CASO ITALIANO: SALVARE IL PLURALISMO E SUPERARE LA POLARIZZAZIONE
}

\author{
di Giovanni Sartori
}

Luciano Pellicani si chiede, e chiede, se l'Italia sia ancora un sistema di «pluralismo polarizzato ${ }^{1}$. Non saprei pensare, con $\mathrm{i}$ tempi che corrono, a un interrogativo di maggiore momento. $\mathrm{E} \mathrm{mi}$ sento in dovere di rispondere subito e per primo. Subito perché gli eventi incalzano e siamo all'ora della verità; e per primo non tanto perché io sia il padre del modello del pluralismo polarizzato, ma per rilanciare il dibattito in seconda battuta, e cioè per promuovere un piú largo dibattito. Ogni terapia presuppone una diagnosi, e cioè una chiara ed esatta percezione dei mali che ci affliggono. E per avere idee chiare occorre discuterle e confrontarle.

Nella prima parte del suo intervento Pellicani si propone di smussare l'antitesi tra la tesi del bipartitismo imperfetto e la tesi del pluralismo polarizzato per ricavarne una «sintesi fertile». L'antitesi - sospetta Pellicani - è dettata piú che da altro da questo sottinteso politico, o pratico: «salvare il sistema attuale o, che è lo stesso, non correre rischi onde evitare il peggio ». E questo perché io sarei, tanto per cominciare, "un pessimista e un realista ». Se pessimista è chi ritiene che al peggio non c'è mai fine, allora sono pessimista. $\mathrm{Ma}$ un medico che fa un referto di prognosi riservata non è un pessimista; e se poi il paziente muore, si dirà di lui che è stato, purtroppo, buon profeta. Comunque sia, oltre ad essere un pessimista e un realista sono anche un ottimista e un idealista (dico cosí per mostrare quante cose si possono dimostrare con queste etichette) che si ostina a credere nel vero e nel falso e nella possibilità di affrontare $i$ problemi conoscitivi in chiave conoscitiva. Pertanto - e vengo al punto - tra Giorgio Galli e me non esiste alcun animus polemico: il nostro disaccordo è puramente euristico. Non ho mai pensato contro Galli, né Galli contro me. Tantovero che le nostre due interpretazioni sono maturate negli stessi anni su basi

1 Verso il superamento del pluralismo polarizzato?, in questo numero della Rivista, pp. 645 ss. 
diversissime, e davvero l'una indipendentemente dall'altra ${ }^{2}$. Ripeto: la divergenza è di diagnosi. E aggiungo - per sbarazzare ancor meglio il terreno dalle equazioni personali - che la mia diagnosi, se valida, ha la stessa utilità per chi vuole «conservare» come per chi vuole cambiare o anche "rivoluzionare».

\section{L'alternanza non è tutto}

Circoscritto il terreno del dibattito, vengo alla conciliazione suggerita da Pellicani, che si può riassumere in tre asserzioni: i) che il modello del pluralismo polarizzato costituisce una spiegazione genetica, a monte, di quell'esito che Galli chiama bipartitismo imperfetto; ii) che pertanto le due tesi non si contraddicono ma anzi si completano sul «punto chiave », e che iii) questa convergenza mi sfugge in parte per ragioni polemiche (il sospetto che ho già cercato di dissipare), e in parte perché sottovaluto - in contraddizione con le mie stesse premesse - l'importanza dell'alternanza al potere. Se cosí non fosse, conclude Pellicani, dovrei convenire con Galli che la possibilità di ricambio delle élites di governo è l'« essenza » della democrazia.

Vediamo, cominciando dalla contraddizione (che per un loico qual io sono è davvero argomento di tutto rispetto). Intanto, mi è venuto in mente che pur avendo scritto (tra poco saranno vent'anni) un lungo libro sulla democrazia non c'è, in quel libro, nemmeno una riga sull'alternanza al governo. Evidentemente non ho mai ritenuto che l'alternanza fosse l'essenza stessa della democrazia. Avrò torto, non dico di no; ma sul punto la mia coerenza è davvero pervicace. E importa che io spieghi perché. L'alternanza al potere riguarda i meccanismi di ottimizzazione, di maggiore e migliore funzionalità, delle democrazie. Pertanto la ritengo auspicabile, e certo non ne sottovaluto l'utilità. Ma rilevo - allargando il discorso che l'alternanza è consentita da certe strutture sistemiche e non da altre, e constato che le democrazie senza alternanza sono parecchie ${ }^{3}$. Per esempio, la Svezia, la Norvegia, l'Irlanda, il Giappone, l'India sono caratterizzate non solo da assenza di alternanza ${ }^{4}$, ma dalla

$2 \mathrm{Nel}$ mio caso partivo dalle tattiche rimunerative di competizione tra partiti in tutti i possibili sistemi. Guardando alle date, il mio articolo sui Modelli spaziali di competizione tra partiti è nel n. 1, 1965 della «Rassegna Italiana di Sociologia »; ma era già tutto scritto nel mio corso universitario Partiti e sistemi di partito del 1964. E il Bipartitismo imperfetto di Galli usciva nel 1966.

3 Vedi, piú estesamente, il mio capitolo Rivisitando il pluralismo polarizzato, nel vol. curato da F. L. Cavazza e S. Graubard, Il caso italiano, Milano, Garzanti, 1974, pp. 196-200.

${ }^{4}$ In Norvegia dal 1935 al 1965, e poi con interruzioni; in Svezia dal 1932 ad oggi (con interruzione 1951-57); in Irlanda dal 1932 al 1973 (con interruzione 1948-57); in Giappone dal 1955 ad oggi; in India dal 1952 ad oggi. 
presenza di un partito predominante che governa da solo. Il che costituisce una aggravante rispetto al caso della DC, di cui non si può dire nemmeno in senso relativo che esercita un potere «assoluto ", visto che la DC è costretta a governare in coalizione. E allora? Sono quantomeno in debito di una risposta che ancora non mi è stata data.

$\mathrm{Si}$ ribatterà che il mio argomento è valido in sede comparata; ma che il problema pratico e concretissimo è, per noi italiani, proprio quello dell'alternanza. Ma non consento, e questo anche e proprio sul terreno pratico. La mia obiezione «pratica » è duplice: i) che a questo modo puntiamo su una carta irrealistica che non ha plausibilità concreta, e che ii) la mancata alternanza si risolve, in pratica, in un magnifico alibi. Un paese che non è «invertebrato » (Pellicani, attento studioso di Ortega, mi capisce) si sa far valere anche senza alternanza. Noi, invece, diamo tutte le colpe alla assenza di alternanza, e con questo lasciamo inoperose le forme alternative di controllo del potere ${ }^{5}$. Certo: la DC è inamovibile, impunita, corrotta e corruttrice. Occorre l'alternanza per punirla? No. E questa è la fondamentale ragione pratica del mio dissentire: non intendo avallare l'alibi. La nostra stampa, la nostra magistratura, e ancor piú i nostri partiti di opposizione, sono tutti colpevoli di larghissime zone di omissione. Chi ha preso coraggio, lo ha preso tardi: e le bordate sono quasi sempre inficiate da sospetti silenzi, da feticci intoccabili, da vacche sacre. Lo ridico per chi vuol capire: un paese « vertebrato » nel quale la stampa, la magistratura e l'opposizione fanno davvero quel che dovrebbero fare, non sarebbe ridotto al punto di putrefazione al quale siamo ridotti. Prima di tutto, facciamo un esame di coscienza. Dopodiché sono pronto a convenire che l'alternanza ci avrebbe aiutato.

Ad ogni modo, la differenza tra la mia «ponderazione » e quella di Galli resta che per me la mancata alternanza è una caratteristica costitutiva del pluralismo polarizzato ${ }^{6}$ : il che vuol dire che è la conseguenza di altre cause che stanno a monte. Si capisce che, in una catena causale, gli effetti sono a loro volta causa di altri effetti. Ma una causa seconda non è una causa prima. E se la mancata alternanza non è - come ritengo - una causa prima, ne consegue che intervenire sulla medesima è uno sbaglio che rischia di aggravare, invece che di migliorare, lo stato del malato. Come quan-

5 Come puntualizza assai bene Giuliano Urbani, Le armi dei cittadini, in «La Stampa », 9 luglio 1974.

6 Pellicani osserva: "Sartori ritiene che la caratteristica dei sistemi bipolari è che "la rotazione al potere è una aspettativa credibile". Che è quanto dire che l'assenza di tale aspettativa deforma la meccanica... di un sistema democratico » (p. 651). Ma nel suo argomento c'è un salto tra «sistemi bipolari » e «sistema democratico ». Se la rotazione caratterizza i sistemi bipolari, non ne consegue che caratterizzi tutti i sistemi democratici, e ciò̀ quelli che bipolari non sono. 
do per estirpare un bubbone rischiamo di diffondere un cancro. Il che mi riporta al nodo della questione. Non solo l'alternanza non è, per me, un «punto chiave »; ma la antitesi tra Galli e me è di modelli, e cioè di fondo.

\section{Teoria della bicicletta e teoria del triciclo}

Detto in metafora, per il modello bipolare noi siamo una $b i$ cicletta, mentre per il modello tripolare noi siamo un triciclo. Una bicicletta è molto piú agile, e per stare in piedi deve stare in moto. Un triciclo non ha la stessa agilità, e può anche stare fermo. La cosa orripilante è che da venticinque anni né la classe politica né quella intellettuale hanno mai dato mostra di capire questa differenza. Seduti su un triciclo, gli abbiamo chiesto prestazioni da bicicletta; con il bel risultato di sgangherare tutte e tre le ruote. E la metafora vale anche a illuminare il contrasto di fondo tra Galli e me. La teoria del bipartitismo imperfetto ci raffigura come una bicicletta la cui imperfezione risiede nel fatto che una ruota è piú grande dell'altra; dal che discende che la soluzione è di renderle approssimativamente eguali. Per la teoria del pluralismo polarizzato noi siamo, invece, un triciclo; dal che discende che, se gli leviamo la ruota di mezzo, il nostro veicolo non può stare in piedi e il capitombolo è tanto certo quanto inevitabile. Fuori di metafora, e per illustrare in concreto, prendiamo il dolente caso cileno (sul quale avevo già messo l'occhio citandolo tra $\mathrm{i}$ casi di pluralismo polarizzato nel ' 67 , e cioè con il senno di prima).

La catastrofe cilena viene ricondotta, dai piú, a fattori esogeni, quali la CIA, lo strangolamento del capitalismo internazionale, e simili. Pellicani cita, a proposito della «storia come complotto", Popper ${ }^{7}$; ed io sono del tutto d'accordo con Popper e con lui nel lasciare ai complotti il peso che hanno, che è raramente risolutivo. Pertanto passo subito alla mia diagnosi strutturale del caso cileno, qual era prima che i militari entrassero in scena, e quale resta a prescindere dal golpe. Il Cile era da tempo un sistema centrifugo tenuto assieme, in chiave centripeta, dalle elezioni presidenziali. Altrimenti dicendo il «centro » del sistema era costituito dall'istituto della Presidenza. Dal 1938 il Cile ha avuto soltanto due presidenti che non fossero espressi da una alleanza centrista: Alessandri nel 1958, e Allende nel 1970. Ad Alessandri, eletto dalle forze di destra, non fu difficile spostarsi al centro, assolvendo cosí al « ruolo di mediazione» che è connaturale a quel posizionamento. Per Allende, invece, la conversione al centro non era possibile. Pertanto l'elezione di Allende creava il vuoto del centro, e per esso

7 Per Karl Popper la teoria «cospirativa » è una sorta di laicizzazione di quella omerica. 
una centrifugazione a tutti i livelli, da quello elettorale a quello di governo, e come tale incontrollabile. Ergo, a partire dal 1970 il mio modello prevedeva che il collasso della democrazia cilena era inevitabile ${ }^{8}$. Se i militari non fossero esistiti, sarebbero stati sostituiti dalle "armate parallele » dei due fronti in conflitto: il MIR da una parte, e Patria y Libertad dall'altra. Insomma, il Cile insegna quel che avremmo già dovuto capire riflettendo sulla caduta della Repubblica di Weimar (1933) e della Repubblica Spagnola (1936): che in un sistema polarizzato un riallineamento bipolare - la trasformazione del triciclo in bicicletta - porta alla guerra civile, o altrimenti alla sopraffazione (senza ritorno) di un estremo sull'altro ${ }^{9}$.

Mi si chiederà: se tanto il Cile quanto l'Italia sono (erano) sistemi polarizzati e, in concreto, tripolari, come si spiega che il Cile sia caduto mentre l'Italia resta ancora in piedi? Rispondo in breve perché sul punto $\mathrm{mi}$ sono già soffermato altrove ${ }^{10}$. L'andamento centrifugo di un sistema polarizzato si misura al livello elettorale e in sede di "politica visibile». Restano, pertanto, i livelli di vertice, e cioè resta da vedere come si comportano le élites in sede di «politica invisibile ». Il Cile è caduto - dicevo - perché era divenuto centrifugo a tutti i livelli, e cioè anche al vertice. L'Italia ha invece seguito la sua antica vocazione trasformistica: il che equivale a dire che noi rattoppiamo al vertice quel che dilaceriamo alla base. Non è, questo, un gran metodo, o un gran siste$\mathrm{ma}^{11}$; e non ne consegue - come dirò tra poco - che l'Italia sia da considerare una «democrazia consociativa ». Ma ne consegue che il nostro paese non è centrifugo a tutti i livelli. Invece di precipitare, scivoliamo. Con il che siamo arrivati al punto centrale, o comunque al quesito che piú ci preme e tormenta. Stiamo scivolando dentro il tunnel del pluralismo polarizzato, oppure ne stiamo scivolando fuori?

8 Per contro (è importante rilevarlo) alla luce del modello bipolare l'elezione di Allende era da interpretare in chiave positiva, e cioè come l'inaugurazione del meccanismo dell'alternanza tra una sinistra e una destra.

9 Questa interpretazione del caso cileno è sviluppata e documentata nel mio volume Parties and Party Systems: A Framework for Analysis, di prossima pubblicazione (1975) per i tipi della Cambridge University Press, cap. 6.2. Nella stessa chiave, e ancor piú diffusamente, vedi l'ottimo capitolo sul Cile di A. Valenzuela, in Juan Linz, A. Stepan (eds.), Breakdowns and Crises of Democratic Regimes, in corso di pubblicazione.

10 Specialmente ne Il caso italiano, cit., pp. 202-204, e 210-211.

11 Pellicani opportunamente richiama, a questo proposito, il concetto di « sviluppo negativo » elaborato da Riggs (vedi sua nota 26). Io parlo, nello stesso senso, di integrazione negativa. 


\section{Democrazia consociativa, segmentazione e polarizzazione}

Precisiamo meglio. Il quesito non è piú se il modello del pluralismo polarizzato adempie alla funzione euristica che gli compete; il quesito diventa se l'Italia sia uscita dalla casella del pluralismo polarizzato. Mentre le classificazioni e le tipologie sono immote, i casi concreti, e cioè i singoli paesi, si muovono. Domanda: in quale direzione ci siamo mossi o stiamo muovendo? Il triciclo si sta rattoppando, si sta sfasciando, oppure sta diventando una bicicletta?

Comincio dall'ipotesi che il triciclo si stia rattoppando, e cioè dall'ipotesi che l'Italia sia diventata, o stia diventando, una «democrazia consociativa ». La dizione è stata coniata nel '68 da Lijphart in riferimento all'Olanda. In Italia è arrivata da circa due anni: e mentre in Italia sta attecchendo, il paradosso è che Lijphart la sta ritrattando ${ }^{12}$. Ad ogni modo, non credo che questo tipo, o modello, sia in alcun modo applicabile all'Italia. Per avvedersene basta elencare $\mathrm{i}$ paesi che sono stati interpretati sub specie di democrazie consociative. Sono soprattutto l'Olanda, la Svizzera, l'Austria, e il Belgio; con qualche cauta e discussa estensione al Libano e ad Israele. Vedi caso, gli autori che hanno davvero lavorato sul modello «consociativo » si sono ben guardati dall'applicarlo all'Italia. Perché? Forse perché ritengono che le nostre élites si scannino da mattina a sera? No. Tutti sanno che l'Italia è da sempre un paradiso trasformistico, che le nostre élites politiche contrattano e barattano, e che sono da tempo altamente «consociate » in omertà, nel lavarsi una mano con l'altra. Dunque, se l'Italia non è stata presa in considerazione una ragione ci deve essere. Difatti c'è: ed è che il modello consociativo si riferisce alle società segmentate, non alle società polarizzate. La Svizzera e il Belgio sono società polietniche, l'Olanda è multi-confessionale e l'Austria è il paese che ha inventato e praticato per 20 anni consecutivi la " grande coalizione » (che è quella tra cattolici e socialisti, con buona pace di chi riferisce ad orecchio). Tutti questi paesi sono sí segmentati o « compartimentalizzati »: ma il loro spazio ideologico destra-sinistra è corto. Difatti in questi paesi i partiti anti-sistema sono del tutto assenti. Dal che consegue, tra l'altro, che la loro struttura non è tripolare. Dove sono, allora, gli isomorfismi, o quantomeno le analogie che consentono l'estensione del modello consociativo all'Italia? Non riesco a vederne nemmeno mezza. Ripeto: una società segmentata pone tutt'altri problemi ed è tutt'altra cosa

12 Vedi A. Lijphart, The Politics of Accomodation: Pluralism and Democracy in the Netherlands, University of California Press, 1968. L'A. prende le distanze dal proprio modello dichiarandolo poco applicabile alla stessa Olanda nell'ultimo capitolo aggiunto alla nuova edizione che uscirà nel 1975 . 
da una società polarizzata. E se «consociazione» sta soltanto per dire - travasando vino vecchio in una botte di moda - che le élites sono, in ultima analisi, solidali e che trattano e raggiungono compromessi, a questa stregua tutte le democrazie che non defungono sono «consociazionali ». Non è una gran scoperta; ed a questo modo abbiamo solo sciupato e vaporizzato uno strumento euristico ${ }^{13}$.

\section{Depolarizzazione e processi di rilegittimazione}

Visto che la diagnosi consociativa ignora o sorvola sulla polarizzazione, è giocoforza rifarsi alla domanda se il nostro è ancora un sistema polarizzato. Domanda alla quale si può rispondere accertando se, e in quale misura, i partiti anti-sistema non siano piú tali. Difatti i partiti anti-sistema stanno ad indicare una distanza ideologica; dal che consegue che di tanto si ammorbidiscono, di altrettanto diminuisce lo spazio ideologico e quindi lo stato di polarizzazione del sistema.

Ma devo premettere, a scanso di fraintesi, due chiarimenti. In primo luogo, non dico « anti-sistema » per contrabbandare una presa di posizione anti-comunista. No: tantovero che nel mio modello il partito anti-sistema della IV Repubblica erano i Gollisti: e questo perché per partito anti-sistema intendo - occorre ripeterlo qualsiasi partito che sia de-legittimante del sistema politico in cui opera. In secondo luogo, non ho mai detto quel che Pellicani mi fa dire, e cioè che il PCI « ha una precisa natura rivoluzionaria che non è cambiata affatto » (p.657). E siccome non l'ho mai detto ${ }^{14}$, non mi si può chiedere di fornire ragioni «positive » di un giudizio - quello sulla natura "rivoluzionaria » del PCI — che non ho mai dato. Quel che dico è che i giudizi dati da altri - e ora anche da Pellicani - sulla trasformazione del PCI non sono fondati su prove adeguate e probanti. Per confortare questa asserzione indico poi quale sarebbe un metodo di prova valido e convincente. E siccome nel mio mestiere il discorso metodologico è importante, è bene che io mi ci soffermi.

Prove che non sono probanti. Non sono tali, dicevo, le interviste ai capi o la conoscenza personale. Questi indicatori non solo

13 Per i necessari approfondimenti debbo rinviare a Parties and Party Systems, cit., cap. 6.3: Moderate Pluralism and Segmented Societies. Per la bibliografia ed una selezione dei vari scritti in argomento, vedi da ultimo Kenneth D. McRae (ed.), Consociational Democracy: Political Accomodation in Segmented Societies, Toronto, McClelland \& Stewart, 1974.

14 Nel Caso italiano, cit., dico anzi l'esatto contrario: «Se mai [il PCI] fu rivoluzionario, non lo è piú " (pp. 210-11). Né esiste alcun passo di un mio qualsiasi scritto nel quale ho mai dichiarato il PCI un partito « rivoluzionario ». 
sono sospetti per ragioni del tutto ovvie, ma sono comunque inficiati da «impressionismo ». Meglio, semmai, studiare la produzione legislativa, come hanno fatto Predieri e Cazzola ${ }^{15}$. Cosí Cazzola rileva che in commissione il duello esistenziale non c'è mai stato, e constata che "quasi i tre quarti della legislazione italiana prodotta tra il 1948 e il 1968 hanno trovato consenzienti i comunisti $»^{16}$. Ma l'indicatore è quello che è, e cioè ha i limiti che ha. In primo luogo investe soltanto il partito parlamentare, e quindi ignora il partito elettorale e anche, per cosí dire, il partito-partito (il che non è dir poco). In secondo luogo il partito parlamentare lo ritroviamo quasi sempre in commissione, e cioè in sede di politica invisibile. In terzo luogo, resta da fare (non certo per colpa di Cazzola, che è arrivato sin dove poteva) una analisi del contenuto. Eppure è soltanto una analisi sistematica e ad boc del contenuto che ci consente di valutare se l'integrazione di un partito antisistema sia negativa oppure positiva e/o in transito da negativa a positiva. Infine, e in quarto luogo, il conto non si può fare soltanto sulle decisioni; deve anche essere fatto sulle non-decisioni. Al quale ultimo proposito Pellicani obietta che qualsiasi governo, se saggio, sconta in anticipo le reazioni dell'opposizione. Certo. Ma il punto è che tanto maggiori sono $\mathrm{i}$ settori di non-decisione nei quali $\mathrm{i}$ governi subiscono il veto degli oppositori, tanto maggiore è l'ingovernabilità o comunque lo stato di atrofia e di inefficienza di un sistema politico. E non c'è dubbio sulla constatazione fatta da Predieri (e da nessuno contestata, che io sappia) che, in Italia, le decisioni importanti non vengono mai, o quasi mai prese ${ }^{17}$.

Passo alle prove che sarebbero probanti di cui lamentavo la carenza. Preciso: probanti se riteniamo che il passaggio da uno stato di polarizzazione ad uno stato di depolarizzazione resta incompiuto e altamente precario finché non avviene a livello democratico, e cioè nei grandi pubblici e nell'arena della politica visibile. A quest'effetto suggerivo una analisi sistematica dei messaggi di comunicazione di massa nell'ottica che si diceva: per accertarne e misu-

15 Alberto Predieri (a cura di), Il processo legislativo nel Parlamento italiano, in corso di pubblicazione per i tipi di Giuffrè; Franco Cazzola, Consenso e opposizione nel Parlamento italiano: il ruolo del PCI, in «Rivista Italiana di Scienza Politica », 1, 1972. Per alcune riserve su quest'ultimo studio cfr. Antonio Lombardo, Comunisti a Ovest di Mosca, in "Gli Stati », ottobre 1974, specie pp. 14-15. Lombardo discute anche tutto il problema degli indicatori.

16 Loc. cit., p. 92.

17 Secondo Pellicani, il PCI non ha la consistenza numerica per esercitare un potere di veto. Il che non toglie che, in pratica, e proprio in virtú del principio delle reazioni anticipate, il rinvio di un provvedimento in aula è quasi equivalente al suo affossamento. Quanto a Predieri il giudizio surriferito è espresso in piú sedi (a cominciare dal suo contributo al vol. coll. Il Parlamento italiano 1946-1963, Napoli, ESI, 1963); ma vedine la migliore documentazione nel vol. cit. supra. 
rarne la riconversione legittimante. Il suggerimento è piaciuto - mi par di capire - a Giacomo Sani, che ha anche trovato la scorciatoia giusta. Quali che siano i messaggi (le ricerche relative sono tutte da fare) Sani si è detto: accertiamone gli effetti. Ed ha ripreso in mano i sondaggi di opinione compiuti da lui stesso e da Barnes guardando a come vengono «percepiti » $\mathrm{i}$ partiti anti-sistema e, viceversa, a come gli elettori di questi partiti percepiscano il sistema ${ }^{18}$. Non mi dilungo su quanto Sani accerta, anche perché confido di poter sollecitamente pubblicare la sua indagine. Basti riferire, in brevissimo, che al 1972 il paese reale (non quello immaginato da chi non guarda mai nessun dato) restava altamente « polarizzato », e cioè plasmato dai messaggi de-legittimanti dai quali era stato allevato. Anticipo solo due dati: il PCI non è «valutato positivamente » nemmeno dalla metà degli elettori del PSI; e nel 1972 solo il $16 \%$ dell'elettorato DC vedeva con favore una coalizione di governo con i comunisti.

Alla luce di queste premesse di metodo, e piú precisamente di verifica, veniamo alla sostanza, e cioè alla tesi (ipotesi) di Pellicani, che si riassume nella seguente asserzione: «il PCI, a partire grosso modo dal 1968, è entrato in una fase di progressiva 'socialdemocratizzazione' »(p. 658). Immagino che Pellicani dica «socialdemocrazia » perché - esperto come è di marxismo - sa bene che di «socialismi » ce ne sono parecchi, ivi incluso quello senza « volto umano ». Ma la sua esigenza di chiarezza è un'arma a doppio taglio. Parlare di socialdemocratizzazione del comunismo è quantomeno esagerare. Dal che consegue che egli si trova in grosse difficoltà con i tempi. Talvolta il suo discorso è al presente, talvolta al futuro prossimo, e talaltra al futuro remoto. Talché la sua esigenza di chiarezza si intride di ambiguità. Pellicani asserisce: «credo che oggi non si possa piú considerare il PCI un partito anti-sistema ». Ma nella riga che segue il discorso si sviluppa già al futuro: «Certo... il processo di integrazione della subcultura comunista è iniziato solo recentemente. $\mathrm{E}$ un partito come il PCI... non diventa 'altro' nel giro di pochi anni » (p. 663). Quanti sono pochi anni? Oggi il PSI è bloccato dagli stessi problemi, lacerazioni e incertezze che ebbe di fronte alla lunga e drammatica crisi che intercorse tra il primo e l'ultimo ministero Facta. Tolte le date, i riferimenti e i nomi propri, una istantanea del socialismo italiano nel 1922 potrebbe essere sovrapposta a quella del PSI nel 1974, e le posizioni di allora risulterebbero pressoché indistinguibili da quelle di oggi. Chi ritiene necessario entrare al governo è Turati o De Martino? Modigliani che illustra con rincrescimento a Bonomi, nel luglio 1922,

18 G. Sani, Mass Perceptions of "Anti-System" Parties: The Case of Italy, Relazione al Congresso della Associazione Americana di Scienza Politica, agosto 1974 . 
che i socialisti gli potevano assicurare solo un appoggio esterno e condizionato, in cosa si distingue dal Mancini dell'ottobre 1974? E a chi attribuire la tesi del rifiuto di principio: stanno parlando i seguaci di Ferri e Serrati, oppure Riccardo Lombardi? Insomma, è passato mezzo secolo e cinquant'anni non sono bastati a socialdemocratizzare il PSI. Quantomeno, nell'ora del pericolo e delle vere assunzioni di responsabilità i socialisti di oggi non sono lontani dal punto in cui erano nel 1922. Inciso a parte, dopo aver asserito che un partito comunista non diventa «altro » in pochi anni, Pellicani passa giustamente a ricordare le difficoltà, $e$ in particolare questa condizione ostante: "In effetti - egli scrive - quello che rende estremamente difficile il passaggio da una integrazione negativa a una integrazione positiva della subcultura comunista è il legame organico del PCI con l'Unione Sovietica » (p. 669). A questo punto, mi sembra, siamo nel futuro remoto. Un legame « organico » non viene meno in pochi anni, ma con il volgere delle generazioni. Torno a chiedere: e allora?

Allora io direi cosí. Nella misura in cui Pellicani disegna un progetto ottimale, o un programma ideale, il suo disegno è anche il mio. Come evidenziato nel titolo, il problema italiano io lo vedo racchiuso tra queste forche caudine: occorre salvare il pluralismo in attesa che, e attendendo a che, venga meno la polarizzazione. Il disaccordo è, quindi, sui tempi e sulle vischiosità che il fattore temporale comporta. Insomma, il tempo è la variabile decisiva. Anni fa la mia prognosi si riassumeva in questa frase: "resta da vedere se... è piú longevo il sistema oppure l'anti-sistema $"{ }^{19}$. Ho sempre dato per scontato, dunque, che le cose si muovono e che i partiti cambiano. Nel Caso italiano precisavo (a proposito del PCI) che " qualsiasi partito si 'accomoda', dopo 25 anni, nel sistema con il quale interagisce. Ma le variazioni trasformistiche non sono tali da poter essere registrate dalla mia classificazione ${ }^{20}$. E qui arriviamo a un altro punto da chiarire.

La classificazione in questione è la tripartizione tra partiti antisistema, partiti di semi-accettazione e partiti pro-sistema. Ora, è di tutta evidenza che uno schema a tre sole voci non può essere uno schema «sensibile ». Con tre sole classi a disposizione, una riclassificazione richiede un salto, una grossa variazione. Pertanto negare la riclassificazione del PCI non equivale in alcun modo a negare che sia cambiato e stia vieppiú cambiando ${ }^{21}$. Equivale semmai

19 Tipologia dei sistemi di partito, in «Quaderni di Sociologia», 3, 1968, p. 219.

20 Op. cit., p. 214.

21 Vale ricordare che nego anche la riclassificazione del MSI, lasciato anch'esso tra i partiti anti-sistema. Non sollevo il problema perché Pellicani non lo tocca; ma alla stregua delle « prove » da lui addotte per dimostrare l'integrazione del PCI risulterebbe, in buona logica, che il MSI è integratissimo. Che è quanto nego appunto perché non accetto quelle prove. 
a sottolineare i limiti di tutti gli schemi comparati, che non possono essere fatti su misura e che peccano necessariamente di troppa capienza. Ma che il limite sia nella poca sensitività dello schema non toglie che anche il cambiamento possa essere misurato male. Molto dipende dal punto di riferimento, o di « azzeramento ». Pellicani si rifà addirittura al chiliasmo e ad un PCI che sarebbe stato rivoluzionario e leninista. Per essere sicuro di non fraintenderlo come, sul punto, lui ha frainteso me, torno a citarlo: «se il PCI fosse ancora un partito anti-sistema, cioè un partito animato dal progetto ...di instaurare una dittatura rivoluzionaria, avrebbe potuto e dovuto scatenare una lotta frontale... » (p. 662). Come si torna a vedere, Pellicani abbatte un po', nel mio caso, un uomo di paglia. Non solo « anti-sistema » non vuol dire « rivoluzionario », ma nella fattispecie né io né i piú hanno mai ritenuto che il PCI plasmato da Togliatti fosse un partito rivoluzionario e leninista. Ragion per cui Pellicani misura, temo, una entità di spostamento che non c'è mai stata.

Aggiungo, a titolo di ulteriore chiarimento, che ogni strumento euristico ha la destinazione che ha. Nel caso in oggetto, la classe dei partiti anti-sistema è intesa a fotografare lo stato di un sistema politico alla base, non ai vertici; e questo per ragioni sulle quali mi sono soffermato in altra sede. Pertanto la Bad Godesberg «silenziosa » di cui scrive Ripa di Meana non può essere recepita dal mio schema: ai fini della depolarizzazione occorre che parli, e che la voce risulti efficace al livello dei processi di rilegittimazione. Pellicani scrive, di conserva, che il PCI ha abbandonato da anni l'alternativa marxista-leninista (rappresentata oggi dai true believers del Manifesto) «quasi furtivamente ». Appunto: una riconversione di rotta pressoché furtiva ci rinvia ai tempi lunghi. Troppo lunghi, e quindi troppo lenti, perché sia già tempo per me di registrarla.

\section{Ricapitolazione}

Possiamo ora tornare alla domanda che piú di ogni altra ci preme: se l'Italia non sia piú, o sia sempre meno, un caso di pluralismo polarizzato. Premetto che come cittadino sarei ultralieto (e dico poco) di rispondere, con Pellicani, che nel caso italiano « $\mathrm{i}$ caratteri tipici del pluralismo polarizzato sono oggi alquanto sbiaditi ». $E$ deve essere altrettanto chiaro che come studioso la cosa non mi disturba affatto: non è che se l'Italia sopravvive il mio modello muore. Il modello aiuta a spiegare (oltre l'Italia) i casi di Weimar, della Repubblica Spagnola, della IV Repubblica, e del Cile. Non è poco, visto che abbraccia tutti i maggiori paesi dell'Europa e il paese piú significativo - in termini di consolidamento democratico - dell'America Latina. E non è tutto: ché il modello del plu- 
ralismo polarizzato include (Italia ora a parte) anche un caso in ottima salute, e cioè di effettiva tenuta centripeta sulle tendenze centrifughe: la Finlandia ${ }^{2}$. Dunque, nulla mi vieta - sub specie di vanità intellettuale - di sottoscrivere questa conclusione: che l'Italia è come la Finlandia. Il mio onore di profeta non è minimamente in gioco, visto che ho sempre e soltanto detto che i sistemi di pluralismo polarizzato tendono all'immobilismo, sono caratterizzati da coalizioni scollate e inefficienti, e che sono fragilissimi se e quando arrivano delle crisi esogene o comunque esplosive. Il che mi pare anche troppo bene confermato - ahimè - da un anno a questa parte, e cioè da quando gli sceicchi hanno messo in crisi tutto il sistema economico internazionale.

Nulla mi vieta, dunque, di allinearmi. Nulla, salvo che non digerisco la superficialità e il sublime disprezzo dei dati - non cercati, oppure bellamente ignorati quando ci sono - che caratterizza tutta la discussione. L'Italia si può salvare solo se diventa, tanto per cominciare, un paese serio. E c'è ben poco di serio - ovviamente abbrevio e generalizzo - nel chiedere, come chiediamo, ai comunisti di salvare la democrazia e, nella fattispecie, una democrazia screditata e decotta. Io mi sono divertito (dico per dire, perché in verità non mi sono divertito per niente) a ipotizzare uno «scenario» di quel che avverrà il giorno in cui il PCI sale al governo, e cioè si trova a dover gestire il « compromesso storico ». La morale del mio futuribile è che il peggior servizio che possiamo rendere al PCI raffigurato da Pellicani e da molti altri (« liberale » che non lo sa, « socialdemocratico » in pectore, e simili) è di metterlo alla prova nelle condizioni che stanno maturando ${ }^{23}$. Pellicani profetizza tensioni decrescenti. Ma da quando il mondo è mondo una crisi economica come quella che ci sta galoppando addosso aumenta e radicalizza le tensioni. E nemmeno i comunisti hanno la bacchetta magica per un paese che si avvicina a grandi passi alla fame (la bilancia dei pagamenti è quella che è) e che, grazie anche alla «tenuta » del PSI, rischia di arrivare ad una inflazione di tipo cileno (il cui tasso si aggirava, nel settembre 1973, attorno al 325 per cento). Possiamo senz'altro dare atto a Berlinguer Amendola e Lama di perseguire una politica «tesa a puntellare il nostro vacillante regime democratico ». Ma anche Berlinguer eredita quel che i suoi predecessori hanno seminato, e cioè una democrazia delegittimata. Se vogliamo salvare il pluralismo - ripeto - occorre superare la polarizzazione. $\mathrm{Ci}$ vuole tempo; $\mathrm{i}$ tempi sono purtroppo infausti; e soprattutto le difficoltà non si superano continuando a sfogliare (ma ormai siamo

22 Sul punto debbo ancora rinviare al vol. cit. Parties and Party Systems, cap. 6.2.

$23 \mathrm{Nel}$ fascicolo sul «Compromesso Storico» di Biblioteca della Libertà, n. 51, luglio-agosto 1974, pp. 92-98: I Comunisti al Governo, E Dopo? 
all'ultima pagina) il libro dei sogni, e cioè facendo finta di non vederle ${ }^{24}$.

Riassumo e concludo. Il primo punto investiva la differenza tra Galli e me, e il proposito di ricavarne una sintesi «fertile». Ma temo che, nella fattispecie, l'antitesi sia piú fertile della sintesi. Difatti la differenza è di modelli, e come tale si riflette in proposte di soluzione radicalmente diverse. Galli propone un riallineamento che produca alternanze, mentre io faccio presente che questa medicina l'abbiamo rivista, da ultimo, in Cile, con l'esito catastrofico che tutti sanno.

Il secondo punto investiva il quesito se l'Italia sia entrata nel novero delle democrazie « consociative »; e qui ribatto, in breve, che se questo modello si applica alla Svizzera e ai paesi segmentati, certo non vale per l'Italia e i paesi polarizzati. E siccome c'è il rischio che la democrazia consociativa diventi un vezzeggiativo per edulcorare il compromesso storico, allora aggiungo che nel mio futuribile prevedo che un governo cattolico-comunista non potrà avere un decorso consociativo.

Il terzo punto era se l'Italia sia uscita dalla casella del pluralismo polarizzato. Quesito al quale rispondo sottolineando che siamo ancora, a mio avviso, un triciclo; beninteso, un triciclo scassatissimo, ma pur sempre un triciclo che riesce a stare in piedi perché e finché tale. Per quanto la polarizzazione sia diminuita (di un quanto che sinora soltanto Sani ha cercato di misurare), è ben certo che non è diminuita al punto da consentire una trasformazione bipolare di un sistema che resta tripolare. Né si può escludere - anche alla luce della crisi che investe tutto l'Occidente - che la polarizzazione torni ad aumentare.

24 A cominciare dalle difficoltà di Berlinguer e del PCI nel suo complesso. Gli uomini di penna fanno presto a dire e a consigliare. Ma Pascal (ne Les Provinciales, Paris, ed. Flammarion, p. 23) faceva rispondere cosí al suo interlocutore in difficoltà: Vous en parlez bien à votre aise. Vous êtes libre et particulier; je suis religieux et en communauté: n'en savez-vous pas peser la différence? 